\title{
Trends in atrial fibrillation ablation: have we maximized the current paradigms?
}

\author{
Roger A. Winkle • R. Hardwin Mead • Gregory Engel • \\ Melissa H. Kong • Rob A. Patrawala
}

Received: 19 November 2011 / Accepted: 28 December 2011 /Published online: 28 February 2012

(C) The Author(s) 2012. This article is published with open access at Springerlink.com

\begin{abstract}
Purpose The purpose of this study was to evaluate how atrial fibrillation (AF) ablation has evolved over time with regards to patient characteristics, procedural variables, complications, and outcomes.

Methods We evaluated trends over time from 2003 to 2010 in clinical characteristics, procedural variables, complications, and Kaplan-Meier AF-free rates after the initial and final AF ablation in 1,125 patients undergoing 1,504 ablations.

Results Evaluating trends from 2003 to 2010, we found that patients undergoing AF ablation became older $(P<0.0001)$, had higher $\mathrm{CHADS}_{2}$ scores $(P<0.0001)$, and more coronary artery disease $(P=0.021)$, persistent AF $(P<0.0001)$, hypertension $(P<0.0001)$, and previous strokes/transient ischemic attacks $(P=0.005)$. Procedure times decreased from $256 \pm 49$ to $122 \pm 28 \mathrm{~min}(P<0.0005)$, fluoroscopy times decreased from $134 \pm 29$ to $56 \pm 19 \min (P<0.0005)$, and major $(P=$ $0.023)$, minor $(P=0.023)$, and total complications $(P=$ $0.001)$ decreased over time. The learning curve to minimize complications was 6 years. For paroxysmal AF, initial ablation AF-free rates improved over time $(P=0.015)$ but improvement plateaued in recent years. For persistent AF, initial ablation AF-free rates trended toward improvement over time $(P=0.062)$ but also plateaued in recent years. For longstanding persistent AF $(P=0.995)$, there was no outcome
\end{abstract}

R. A. Winkle $(\bowtie) \cdot$ R. H. Mead $\cdot$ G. Engel $\cdot$ M. H. Kong $\cdot$

R. A. Patrawala

Silicon Valley Cardiology,

1950 University Avenue, Suite 160,

East Palo Alto, CA 94303, USA

e-mail: rawinkle@aol.com

R. A. Winkle $\cdot$ R. H. Mead - G. Engel $\cdot$ M. H. Kong •

R. A. Patrawala

Sequoia Hospital,

Redwood City, CA, USA improvement after initial ablation over time. There was no trend for improved final outcomes (including repeat ablations) over time for paroxysmal, persistent, or long-standing AF ( $P=$ 0.150 to $P=0.978$ ).

Conclusions Despite decreased procedural and fluoroscopy times and reduced complication rates, post-ablation freedom from AF has not improved commensurately in recent years. A better understanding of AF initiation and maintenance may be required to devise personalized approaches to AF ablation and further improve outcomes.

Keywords Atrial fibrillation · Ablation · Outcomes

\section{Introduction}

The discovery of pulmonary vein (PV) tachyarrhythmias as focal triggers of atrial fibrillation (AF) in many patients resulted in catheter ablation as a treatment modality for AF [1]. AF initiation and maintenance has also been attributed in some patients to non-PV sites like the posterior left atrial (LA) wall, ligament of Marshall, superior vena cava, coronary sinus [2], vagally mediated LA ganglionated plexi (GP) $[3,4]$, and areas of complex fractionated atrial electrograms (CFAE) [5] and rotors [6]. Despite the recognized contribution of non-PV drivers to the initiation and maintenance of $\mathrm{AF}, \mathrm{PV}$ isolation remains the gold standard for ablation aimed at eliminating symptomatic AF [7]. Studies reporting ablation outcomes show that persistent or long-standing persistent AF, LA size, and the presence of hypertension, diabetes, coronary artery disease, and non-PV triggers decrease the success of AF ablation [8-16]. Single procedures do not always eliminate $\mathrm{AF}$ and repeat ablations are an important part of the AF ablation strategy [8]. Despite these advances, many patients undergo AF ablation without elimination of their AF 
[17]. The present study examines a single center's experience with $\mathrm{AF}$ ablation and our changes over time in patient demographics, outcomes of initial and final ablations, procedural variables, and complications. These temporal trends provide insights into the evolution of $\mathrm{AF}$ ablation and suggest that ablationists may need a better understanding of individual patients $\mathrm{AF}$ mechanisms to further improve $\mathrm{AF}$ ablation outcomes.

\section{Methods}

\subsection{Patient population}

The subjects were consecutive symptomatic patients undergoing AF ablation at Sequoia Hospital, Redwood City, CA, USA from October 10, 2003 to December 31, 2010. All signed written informed consent. Data collection was prospective and approved by the hospital IRB. AF type was categorized as paroxysmal (AF1: lasting $<1$ week), persistent (AF2: lasting $>1$ week and $<1$ year or requiring pharmacological or electrical cardioversion in $<1$ week), and long-standing persistent (AF3: lasting $>1$ year) [7].

\subsection{Ablation protocol}

Our ablation protocol [8] and anticoagulation strategy [18] have been previously published, and some of the patients in this study were included in previous publications [8, 19]. Antiarrhythmic drugs (AADs) were stopped $\geq 5$ half-lives and amiodarone $\geq 3$ months before ablation. General anesthesia and right groin venous access were used in most ablations. A $7 \mathrm{~F}$ duodecapolar catheter (St. Jude (St. Paul, MN, USA) Livewire ${ }^{\mathrm{TM}}$ ) was placed around the tricuspid valve annulus with the distal poles in the coronary sinus. A $9 \mathrm{~F}$ Boston Scientific (Natick, MA, USA) Ultra Ice ${ }^{\mathrm{TM}}$ catheter guided the transseptal puncture, done using a $71-\mathrm{cm}$ St. Jude $\mathrm{BRK}^{\mathrm{TM}}$ or Baylis (Montreal, QC, Canada) NRG ${ }^{\mathrm{TM}}$ needle [20]. Patients had a femoral or radial arterial line. The St. Jude NavX ${ }^{\mathrm{TM}}$ system was used in all cases. Prior to January 2006, we used a closed tip catheter (Boston Scientific Blazer II $^{\mathrm{TM}}$ or Webster (Diamond Bar, CA, USA) Celsius ${ }^{\mathrm{TM}} 8 \mathrm{~mm}$ ) and, thereafter, an open irrigated tip catheter (Webster Thermocool $^{\mathrm{TM}} 3.5 \mathrm{~mm}$ or St. Jude Cool Path ${ }^{\mathrm{TM}}$ or Sapphire$\left.\mathrm{Blu}^{\mathrm{TM}} 4.0 \mathrm{~mm}\right)$. Most irrigated tip catheter ablations were done using $50 \mathrm{~W}$ and the technique of "perpetual motion" where the catheter is moved back and forth across a small area and not left at single sites for extended times [21]. All patients underwent circumferential atrial ablation around all PVs and a LA roof-line ablation. Only patients with right or LA isthmus flutter underwent a caval-tricuspid or mitral isthmus linear ablation. Many patients had low posterior LA lines and those with LA CFAE had them ablated. Some patients underwent ablation in the coronary sinus (at 30-35 W) and/or superior vena cava isolation. After wide area ablation around all veins, a circumferential mapping catheter $\left(7 \mathrm{~F}\right.$ Webster Lasso ${ }^{\mathrm{TM}}$ or St. Jude Reflexion Spiral ${ }^{\mathrm{TM}}$ ) was used to isolate all PVs defined as complete local electrical silence indicating entrance block. After May 2010, all patients underwent pacing from the PVs to document exit block. Nav $\mathrm{X}^{\mathrm{TM}}$ activation and entrainment mapping were used to ablate flutters and tachycardias. Isoproterenol was given and non-PV triggers were mapped and ablated.

\subsection{Anticoagulation}

Patients receiving warfarin continued it until 5 days preprocedure. Three days pre-procedure, they began enoxaparin $1 \mathrm{mg} / \mathrm{kg}$ every $12 \mathrm{~h}$ with the last dose $24 \mathrm{~h}$ pre-ablation. Patients with AF2, AF3, or frequent AF1 underwent transesophageal echocardiogram. When the transseptal sheath entered the LA, we gave a heparin bolus followed by a $1,000-\mathrm{U} / \mathrm{h}$ infusion through the transseptal sheath adjusted by the activated clotting time (ACT). The initial target ACT was $>300-350$ s, reduced to 225 after the irrigated tip catheter was introduced [18]. Post-ablation, we used enoxaparin $0.5 \mathrm{mg} / \mathrm{kg}$ q12h until warfarin (continued $\geq 3$ months) achieved an international normalized ratio (INR) of 2.0-3.0. After November 2010, some patients received dabigatran following ablation [22].

\subsection{Data collection and analysis}

For each patient, we recorded age, gender, AF duration and type, number of antiarrhythmic drugs failed, $\mathrm{CHADS}_{2}$ score, body mass index (BMI), strokes/transient ischemic attacks (TIAs), LA size, prior cardioversions, and comorbidities including hypertension, diabetes, coronary artery disease, and cardiomyopathy. For each ablation, we recorded average ACT, fluoroscopy, and procedure time. Procedural data were evaluated by ablation year for all initial and repeat ablations combined. For all patients of each AF type, outcome was determined after the initial ablation by year of procedure and after the final ablation by year of procedure. Due to the small number of patients, years 2003 and 2004 were combined.

\subsection{Complications}

Complications were categorized as major or minor by the year of ablation for the combined initial and repeat ablations. Major complications included death, pericardial tamponade, stroke, atrio-esophageal fistula, PV stenosis $>50 \%$ requiring intervention, groin complications requiring surgery or transfusion, heart block, severe tongue hematoma due to intubation, and severe protamine reaction. Minor complications included 
transient cough, elective late pericardiocentesis, groin complications treated with a minor procedure, esophageal irritation requiring no intervention, $\mathrm{PV}$ stenosis $>50 \%$ not requiring intervention, pacer lead dislodgement, TIA, urethral trauma, urinary tract infection, mild tongue hematoma due to intubation, and pneumonia.

\subsection{Follow-up}

Many patients were treated with AADs during the 3month blanking period post-ablation. Patients transmitted daily electrocardiogram (ECG) strips for 1-3 months post-ablation and were seen at 3 months when an echocardiogram and ambulatory ECG monitor for $\geq 24 \mathrm{~h}$ were performed. Initial ablation failures, defined as recurrent $\mathrm{AF}$, tachycardia, or flutter lasting $>30 \mathrm{~s}$ off of drugs $\geq 3$ months post-procedure, were encouraged to undergo a repeat ablation. Patients were seen or contacted frequently from 3-12 months and came for a 1-year followup with echocardiogram and 24-h ECG. Thereafter, every 6-12 months, patients were seen directly or contacted via telephone by research nurses or the attending physician, and arrhythmia records were obtained from hospitals and referring physicians. Patients were advised to call for arrhythmia symptoms and ECG recorders were reissued to them. During 2011, all patients considered AF-free $>1$ year post final ablation were encouraged to undergo a 1-week continuous monitor. Late pacemaker AF data were utilized when available. One patient was lost to follow-up.

\subsection{Statistical analysis}

Statistical analysis was done using XLSTAT 2010 (Paris, France). Continuous data were described as mean \pm standard deviation and categorical data as counts and percentages. Analysis of variance and the Cochran-Armitage trend analysis were used to compare procedural variables including average ACT, procedure and fluoroscopy times, and complications, as well as clinical parameters including age, gender, BMI, AF duration and type, number of AADs failed, prior cardioversions, LA size, $\mathrm{CHADS}_{2}$ score, and the presence of coronary artery disease, dilated cardiomyopathy, hypertension, and prior stoke/TIA among patients grouped by year of initial ablation. Kaplan-Meier curves were generated for AF-free survival by AF type and by ablation year for the initial ablation and for final outcome by the year of the last ablation. All tests were two-sided and $P<0.05$ was considered statistically significant.

\section{Results}

\subsection{Patient population}

The subjects were 1,125 consecutive patients who underwent 1,504 ablations for symptomatic AF. Table 1 shows the patient characteristics by year of initial ablation. The number of initial ablations increased from 40 to 280 from 2003 to 2010 . The total number of ablations/year increased from 43 to 380 . There

Table 1 Clinical characteristics by year of initial ablation

\begin{tabular}{|c|c|c|c|c|c|c|c|c|}
\hline & $2003-2004$ & 2005 & 2006 & 2007 & 2008 & 2009 & 2010 & $P$ value \\
\hline Initial ablations & 40 & 65 & 133 & 170 & 196 & 241 & 280 & NA \\
\hline Repeat ablations & $3(7.0 \%)$ & $14(17.7 \%)$ & $43(24.4 \%)$ & $63(27.0 \%)$ & $71(26.6 \%)$ & $85(26.1 \%)$ & $100(26.3 \%)$ & $=0.036^{*}$ \\
\hline Total ablations & 42 & 79 & 176 & 233 & 267 & 326 & 380 & NA \\
\hline Age (years) & $59.0 \pm 11.1$ & $58.4 \pm 10.5$ & $61.6 \pm 9.9$ & $61.4 \pm 10.6$ & $62.1 \pm 10.3$ & $62.8 \pm 10.0$ & $64.3 \pm 10.0$ & $<0.0001^{*}$ \\
\hline Gender ( $\%$ female $)$ & $35.0 \%$ & $23.1 \%$ & $23.3 \%$ & $30.6 \%$ & $25.5 \%$ & $30.3 \%$ & $31.8 \%$ & $=0.202$ \\
\hline Duration of AF (years) & $5.6 \pm 5.3$ & $7.9 \pm 8.0$ & $6.9 \pm 6.8$ & $6.6 \pm 7.8$ & $5.9 \pm 6.2$ & $6.7 \pm 7.8$ & $6.0 \pm 6.5$ & $=0.336$ \\
\hline \# Drugs failed & $1.55 \pm 0.96$ & $1.58 \pm 1.01$ & $1.41 \pm 1.18$ & $1.22 \pm 1.00$ & $1.15 \pm 0.97$ & $1.25 \pm 0.98$ & $1.34 \pm 1.14$ & $=0.336$ \\
\hline Average $\mathrm{CHADS}_{2}$ score & $0.77 \pm 0.95$ & $0.58 \pm 0.88$ & $0.78 \pm 0.83$ & $0.70 \pm 0.88$ & $0.78 \pm 0.97$ & $0.91 \pm 0.94$ & $1.15 \pm 1.08$ & $<0.0001 *$ \\
\hline Body mass index & $28.1 \pm 4.3$ & $29.2 \pm 5.5$ & $29.5 \pm 5.4$ & $29.5 \pm 5.4$ & $29.3 \pm 5.1$ & $29.5 \pm 5.4$ & $29.9 \pm 5.6$ & $=0.601$ \\
\hline LA size $(\mathrm{cm})$ & $4.13 \pm 0.77$ & $4.36 \pm 0.66$ & $4.37 \pm 0.62$ & $4.38 \pm 0.67$ & $4.31 \pm 0.71$ & $4.26 \pm 0.67$ & $4.29 \pm 0.72$ & $=0.308$ \\
\hline Hypertension & $45.0 \%$ & $24.6 \%$ & $40.6 \%$ & $38.8 \%$ & $46.4 \%$ & $49.0 \%$ & $57.9 \%$ & $<0.0001^{*}$ \\
\hline Diabetes & $10.0 \%$ & $13.8 \%$ & $6.8 \%$ & $7.1 \%$ & $8.2 \%$ & $5.8 \%$ & $12.9 \%$ & $=0.458$ \\
\hline Cardiomyopathy & $2.5 \%$ & $13.3 \%$ & $10.5 \%$ & $9.4 \%$ & $5.1 \%$ & $7.1 \%$ & $8.9 \%$ & $=0.710$ \\
\hline Coronary artery disease & $7.5 \%$ & $10.8 \%$ & $8.3 \%$ & $11.8 \%$ & $15.3 \%$ & $15.5 \%$ & $15.0 \%$ & $=0.021 *$ \\
\hline Prior cardioversion & $45.0 \%$ & $44.6 \%$ & $44.4 \%$ & $47.6 \%$ & $46.4 \%$ & $46.3 \%$ & $48.6 \%$ & $=0.415$ \\
\hline Prior stroke/TIA & $2.5 \%$ & $1.5 \%$ & $8.3 \%$ & $3.5 \%$ & $6.1 \%$ & $7.1 \%$ & $8.9 \%$ & $=0.005^{*}$ \\
\hline
\end{tabular}

$A F$ atrial fibrillation, $T I A$ transient ischemic attack, $N A$ not applicable

$* P<0.05$ 
was a gradual increase in age from $59.0 \pm 11.1$ to $64.3 \pm$ 10.0 years $(P<0.0001)$ and an increase in the average $\mathrm{CHADS}_{2}$ score from $0.77 \pm 0.95$ to $1.15 \pm 1.08(P<0.0001)$. Over time, more patients had comorbidities including hypertension $(P<0.0001)$, coronary artery disease $(P<0.021)$ and prior strokes/TIAs $(P=0.005)$. Although there were more repeat ablations $(P=0.036)$ over time, this largely resulted from the fact that in the early years of our AF ablation program, there were only initial ablations. From approximately 2007 to 2010, the percentage of repeat ablations remained stable at $26 \%$. There was no change in gender, number of AADs failed, duration of AF prior to ablation, BMI, or LA size over time.

There was a gradual change in the composition of patients referred for AF ablation by AF type (Fig. 1). There was a slight decrease in the number of patients with AF1 which did not reach statistical significance $(P=0.07)$, a decline in the number with AF3 $(P<0.001)$ and an increase in the number with AF2 $(P<0.0001)$.

\subsection{Procedural variables}

Figure 2 shows the average procedural and fluoroscopy times for the combination of initial and repeat ablations by year. From 2003-2004 to 2010, there was a significant shortening of procedure times from $256 \pm 49$ to $122 \pm 28 \min (P<0.0005)$ and fluoroscopy times from $134 \pm 29$ to $56 \pm 9 \mathrm{~min}(P<$ 0.0005). Figure 3 shows the decrease in ACT (from $338 \pm 30$ to $229 \pm 21 \mathrm{~s}, P<0.0001)$ over time.

\subsection{Outcomes by year}

Figure 4 shows the Kaplan-Meier curves for freedom from AF after initial ablation by year and AF type. Figure 4(A) shows a statistically significant year-to-year improvement in outcomes after the initial ablation for AF1 patients $(P=$

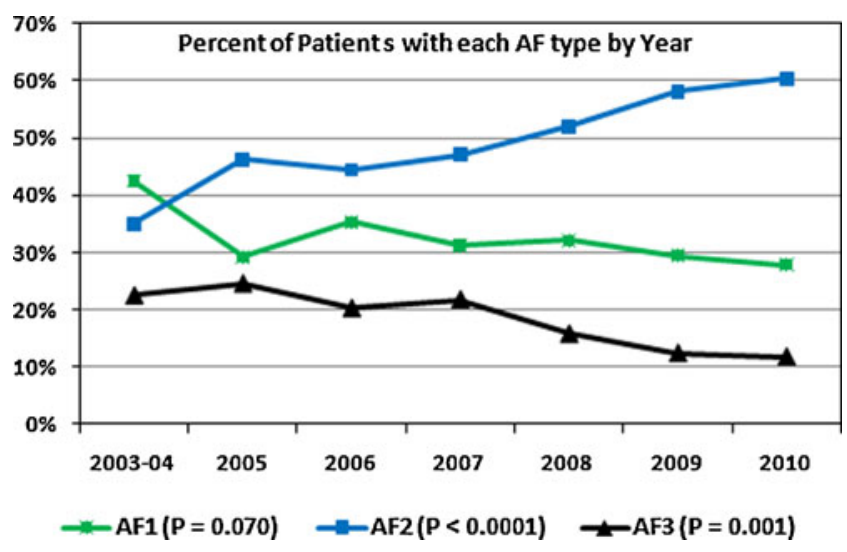

Fig. 1 Percentage of AF patients with each type of AF (green = paroxysmal $\mathrm{AF} 1$, blue $=$ persistent $\mathrm{AF} 2$, and black $=$ long-standing persistent AF3) by year of initial ablation

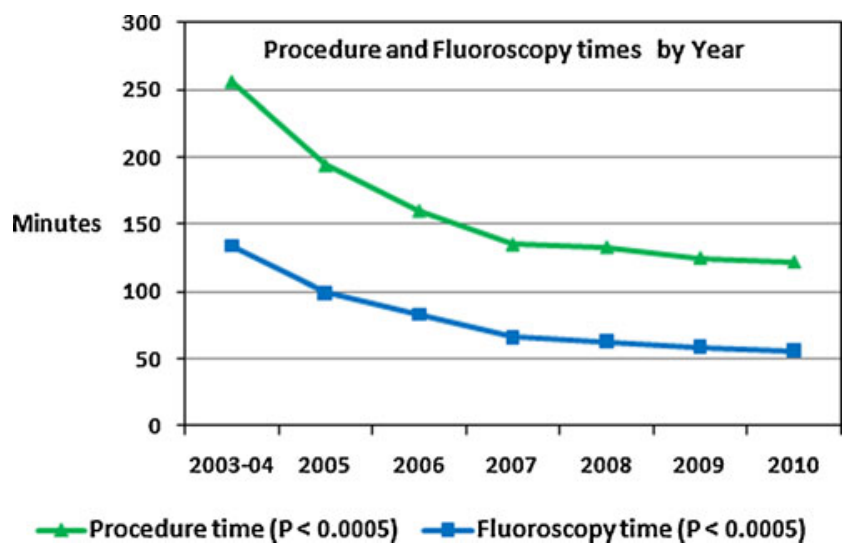

Fig. 2 Changes in procedure and fluoroscopy time (green = procedure time, blue $=$ fluoroscopy time) by year of initial ablation

0.015). Despite the overall improvement, inspection of the Kaplan-Meier curves shows little improvement after 2006. Figure 4(B) shows a trend towards year-to-year improvement in initial ablation outcomes for AF2 patients $(P=0.062)$ with no improvement after 2007. Figure 4(C) shows year-to-year initial ablation outcomes for AF3 patients and demonstrates no improvement over the years $(P=0.995)$.

Figure 5 shows the Kaplan-Meier curves for the outcome after the final ablation (average of 1.34 ablations/patient) by year of last ablation. Figure 5(A-C) shows that there was no improvement over time for the final outcome in patients with $\mathrm{AF} 1(P=0.325), \mathrm{AF} 2(P=0.150)$, or AF3 $(P=0.978)$. For both $\mathrm{AF} 1$ and $\mathrm{AF} 2$, the rates of freedom from $\mathrm{AF}$ after the final ablation for 2010 may be artificially low due to the fact that many of these patients have only undergone an initial ablation.

\subsection{Procedural complications}

Figure 6 shows the trend over time for procedural complications for the combination of initial and repeat ablations by year. Major procedural complications have decreased gradually from $4.65 \%$ of ablations in $2003-2004$ to $0.53 \%$ in 2010

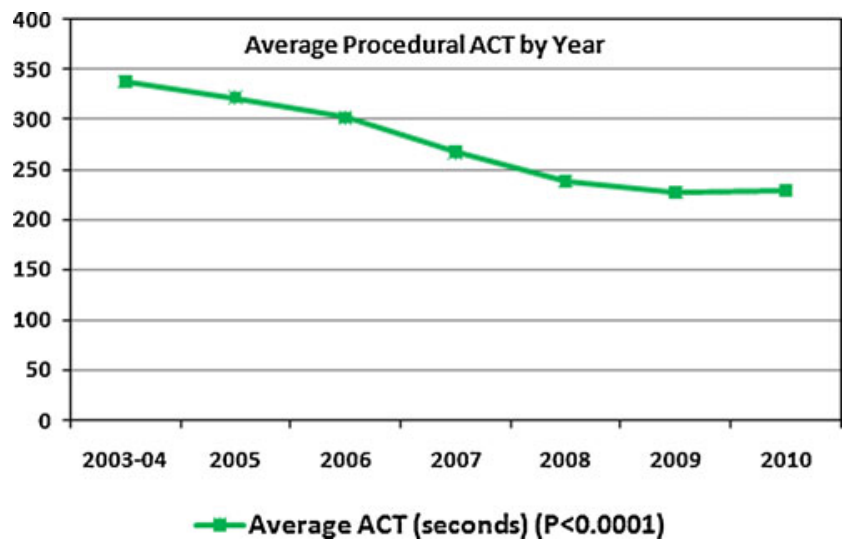

Fig. 3 Decline in average procedural ACT by year 

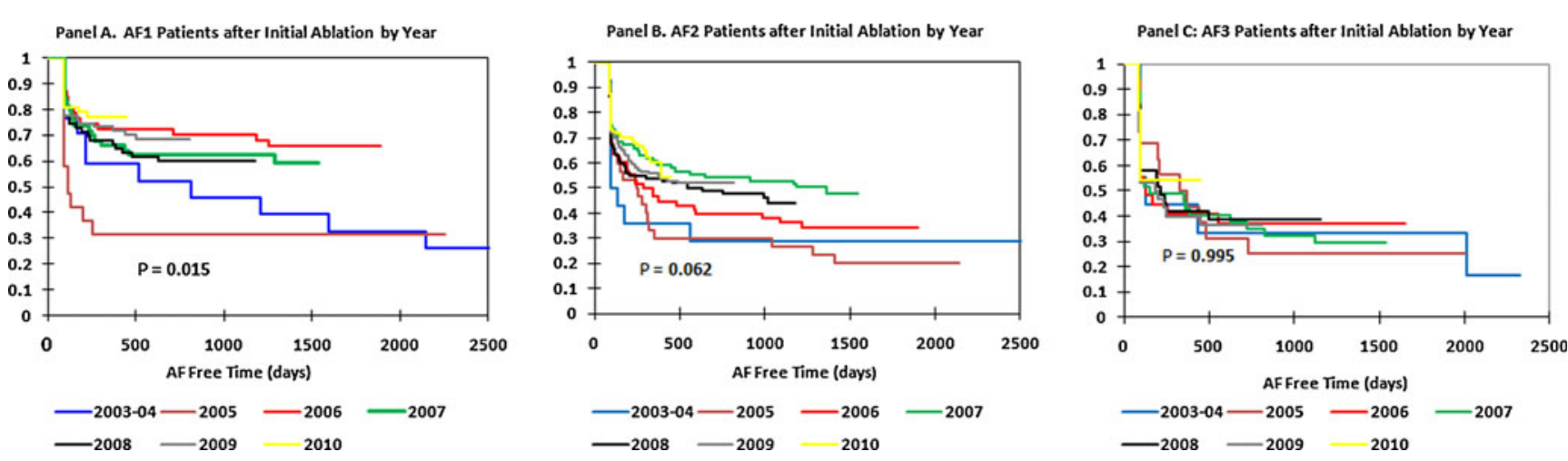

Fig. 4 Kaplan-Meier curves for freedom from AF after initial AF ablation by year ((A) paroxysmal AF1, (B) persistent AF2, (C) long-standing persistent AF3). Blue $=2003-2004$, purple $=2005$, red $=2006$, green $=2007$, black $=2008$, gray $=2009$, yellow $=2010$

$(P=0.023)$. Minor complications also decreased over time from $4.65 \%$ in 2003 and 2004 to $1.05 \%$ in $2010(P=0.023)$. The combined total of major and minor complications decreased from $9.30 \%$ to $1.58 \%$ over time $(P=0.001)$. The shape of the curve indicates a long learning curve of about 6 years to minimize ablation complications. All individual major and minor complications over time are shown in Table 2. There were no deaths, atrio-esophageal fistulae, or PV stenoses requiring intervention. Although the total number of complications decreased over time, the incidence of pericardial tamponade was the only individual complication to decrease over time $(P=0.02)$.

\section{Discussion}

This study reveals two key trends that occurred over time at our institution: (1) the clinical characteristics of patients undergoing $\mathrm{AF}$ ablation changed and (2) post-procedure outcomes did not improve commensurate with improvements in procedural performance. From 2003 to 2010, there was a gradual change in the baseline demographics of patients undergoing AF ablation. Patients are now older with higher $\mathrm{CHADS}_{2}$ scores and more persistent AF. The recognition of these trends is critical because of the key role that clinical factors play in determining AF ablation efficacy. The fact that LA size, duration of AF, number of AADs failed and prior cardioversions did not change over time suggests that patients are not being referred for ablation earlier in their disease process. The outcomes of AF ablation might be improved by earlier referral.

Despite optimization of procedural factors, i.e., performing $\mathrm{AF}$ ablations faster and with fewer complications, our outcomes after the initial and final ablation have either not improved or have plateaued. Improvements in outcomes for initial ablations over time were greatest for AF1 and least for AF3. However, even for AF1, ablation outcomes seem to have reached a ceiling several years ago. Although we appear to have optimized our AF-free rates after a few years of ablation experience, it took approximately 6 years and almost 800 ablations for the complication rates to reach a nadir. This suggests that the learning curve for performing ablations safely is not insignificant even in the hands of experienced ablationists. Part of our reduction in complications may be due to operator experience; however, part is due to procedural changes such as using only one groin for all venous access and the use of the radiofrequency transseptal needle which we
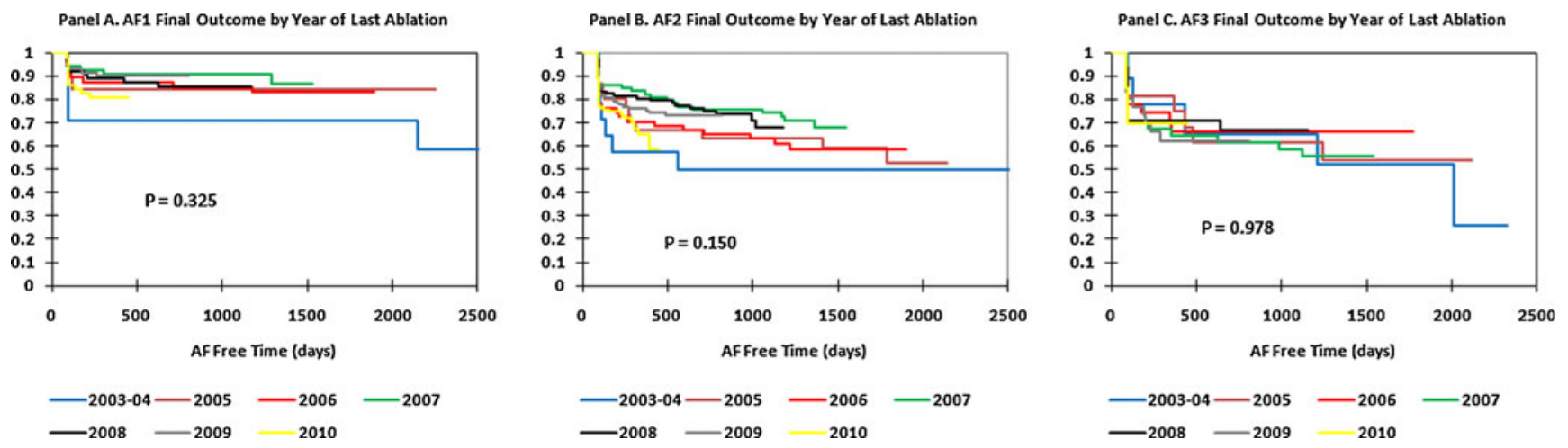

Fig. 5 Kaplan-Meier curves for freedom from AF after the last AF ablation by year of last ablation. (A) Paroxysmal AF1, (B) persistent AF2, (C) long-standing persistent AF3. Color code the same as Fig. 4 


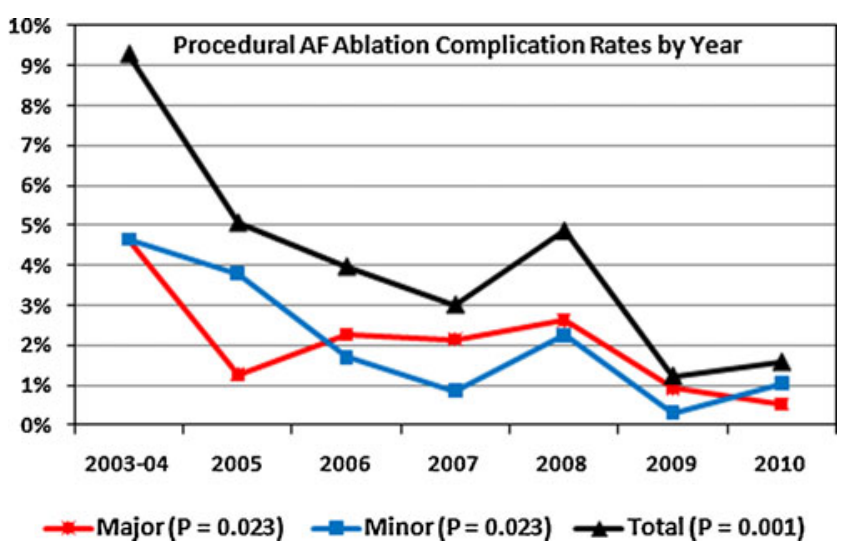

Fig. 6 Percent of AF ablations with major (red) and minor (blue) complications by year. The black line represents the total complications (major + minor) by year

have shown to reduce the incidence of pericardial tamponade [20]. While we recognize that most centers keep their target ACT $>300-350 \mathrm{~s}$, we have found that lowering our target ACT level reduced our overall vascular and hemorrhagic complication rate [18]. It is important to emphasize that when keeping the target ACT lower, one must use only a single transseptal puncture and should infuse maintenance heparin through the transseptal sheath. The change to general anesthesia [23] and the use of the irrigated tip ablation catheter at $50 \mathrm{~W}$ [21] early in our ablation series may also have contributed to procedural improvements over time. Many centers now perform AF ablation on patients with a therapeutic INR.
However, it is becoming more difficult to do uninterrupted oral anticoagulation as more patients are referred for ablation on the direct thrombin inhibitor, dabigitran, which has no reversal agent, making it potentially dangerous to perform a procedure on a fully orally anticoagulated patient [22].

Lemola et al. [24] showed that when an initial ablation has failed, repeat electroanatomic mapping shows that $\geq 1$ PVs have reconnected in $98 \%$ of patients. PV reconnection is not necessarily, however, the only cause of ablation failure. It is also possible that even if we can permanently eliminate all existing PV-LA connections with a single procedure, new connections may regenerate de novo over time. Studies have shown that PV isolation is not necessarily needed to eliminate AF [25]; for example, ablation of CFAEs or left atrial GP can eliminate AF in some patients [3-5]. In a randomized trial of AF1 patients, Katritsis et al. [3] reported that $45.5 \%$ were AF-free with PV isolation alone compared with $73.5 \%$ with PV isolation plus GP ablation at 4.7 months post-ablation. In our series, the wide area circles we ablate around all veins prior to using a circular catheter to finish vein isolation encompass the major anatomical locations of GP, probably accomplishing both PV isolation and GP ablation. Our AF-free rate after a single ablation is virtually identical to those of Katritsis et al. [3] in patients who received both PV isolation and GP ablation. The role of rotors and CFAEs in AF ablation remains incompletely understood. Preliminary evidence suggests that ablating rotors may also alleviate AF [6].

Table 2 Complications by year of initial ablation

\begin{tabular}{lllllllll}
\hline & $2003-2004$ & 2005 & 2006 & 2007 & 2008 & 2009 & 2010 & $P$ value \\
\hline Total ablations & 43 & 79 & 176 & 233 & 267 & 326 & 380 & $\mathrm{NA}$ \\
Cough which resolved & $0(0.0 \%)$ & $0(0.0 \%)$ & $0(0.0 \%)$ & $1(0.4 \%)$ & $2(0.7 \%)$ & $0(0.0 \%)$ & $1(0.3 \%)$ & $=0.22$ \\
Groin complications (major + minor) & $0(0.0 \%)$ & $1(1.3 \%)$ & $4(2.3 \%)$ & $1(0.4 \%)$ & $6(2.2 \%)$ & $1(0.3 \%)$ & $1(0.3 \%)$ & $=0.11$ \\
Esophageal irritation & $0(0.0 \%)$ & $0(0.0 \%)$ & $1(0.6 \%)$ & $0(0.0 \%)$ & $0(0.0 \%)$ & $0(0.0 \%)$ & $0(0.0 \%)$ & $=0.22$ \\
PV stenosis $>50 \%$ no intervention & $0(0.0 \%)$ & $0(0.0 \%)$ & $0(0.0 \%)$ & $0(0.0 \%)$ & $1(0.4 \%)$ & $0(0.0 \%)$ & $0(0.0 \%)$ & $=0.97$ \\
Pacer lead dislodgment & $0(0.0 \%)$ & $0(0.0 \%)$ & $1(0.6 \%)$ & $0(0.0 \%)$ & $0(0.0 \%)$ & $0(0.0 \%)$ & $0(0.0 \%)$ & $=0.22$ \\
Urethral trauma & $0(0.0 \%)$ & $0(0.0 \%)$ & $0(0.0 \%)$ & $1(1.3 \%)$ & $0(0.0 \%)$ & $0(0.0 \%)$ & $0(0.0 \%)$ & $=0.52$ \\
Severe urinary infection & $0(0.0 \%)$ & $1(1.3 \%)$ & $0(0.0 \%)$ & $0(0.0 \%)$ & $0(0.0 \%)$ & $0(0.0 \%)$ & $0(0.0 \%)$ & $=0.07$ \\
Pneumonia & $1(2.3 \%)$ & $0(0.0 \%)$ & $0(0.0 \%)$ & $0(0.0 \%)$ & $0(0.0 \%)$ & $1(0.3 \%)$ & $0(0.0 \%)$ & $=0.19$ \\
Eye keratopathy, transient & $0(0.0 \%)$ & $0(0.0 \%)$ & $0(0.0 \%)$ & $0(0.0 \%)$ & $0(0.0 \%)$ & $0(0.0 \%)$ & $1(0.3 \%)$ & $=0.25$ \\
Tongue hematoma (major + minor) & $0(0.0 \%)$ & $0(0.0 \%)$ & $0(0.0 \%)$ & $0(0.0 \%)$ & $0(0.0 \%)$ & $0(0.0 \%)$ & $2(0.5 \%)$ & $=0.10$ \\
Elective, non-tamponade pericaridocentesis & $0(0.0 \%)$ & $1(1.3 \%)$ & $0(0.0 \%)$ & $0(0.0 \%)$ & $0(0.0 \%)$ & $0(0.0 \%)$ & $0(0.0 \%)$ & 0.07 \\
Pericardial tamponade & $1(2.3 \%)$ & $1(1.3 \%)$ & $1(0.6 \%)$ & $3(1.3 \%)$ & $2(0.7 \%)$ & $1(0.3 \%)$ & $0(0.0 \%)$ & $=0.02^{\mathrm{a}}$ \\
Stroke/TIA & $2(4.7 \%)$ & $0(0.0 \%)$ & $0(0.0 \%)$ & $0(0.0 \%)$ & $1(0.4 \%)$ & $1(0.3 \%)$ & $1(0.3 \%)$ & $=0.15$ \\
Severe protamine reaction & $0(0.0 \%)$ & $0(0.0 \%)$ & $0(0.0 \%)$ & $0(0.0 \%)$ & $1(0.4 \%)$ & $0(0.0 \%)$ & $0(0.0 \%)$ & $=0.97$ \\
Heart block & $0(0.0 \%)$ & $0(0.0 \%)$ & $0(0.0 \%)$ & $1(0.4 \%)$ & $0(0.0 \%)$ & $0(0.0 \%)$ & $0(0.0 \%)$ & $=0.52$ \\
Total & $4(9.3 \%)$ & $4(5.1 \%)$ & $7(4.0 \%)$ & $7(3.0 \%)$ & $13(4.9 \%)$ & $4(1.2 \%)$ & $6(1.6 \%)$ & $=0.001^{\mathrm{a}}$ \\
\hline
\end{tabular}

TIA transient ischemic attack, $N A$ not applicable

${ }^{\text {a }}$ Statistically significant 
Our data also suggest that one cannot use long-term outcomes from patients with paroxysmal AF ablations years ago to judge the efficacy of contemporary ablations. Weerasooriya et al. [26] reported only a $40 \%$ longterm success rate after a single procedure done in Bordeaux, France in 2001-2002. A more contemporary series from that center reported a 1-year AF-free rate of $61.8 \%$ after a single procedure [27].

Many of the new AF ablation technologies may not be able to significantly improve AF ablation results. Robotic [28] and magnetic navigation systems [27] do the job that ablationists do manually with a different technology but do not contribute to a better understanding of what we should be ablating. Although it is theoretically possible that better tissue contact with a robotic system could result in better ablation outcomes, early results do not suggest this is the case. In a study randomizing patients to manual catheter manipulation vs. magnetically controlled ablation, there was no difference in outcome and patient radiation exposure and procedure time increased with the magnetic navigation system [27]. Other technologies holding promise for improved $\mathrm{PV}$ isolation have not yet demonstrated improved outcomes. The cryoballoon is another promising new technology for AF ablation. A recent literature review of the efficacy of this technology showed a single procedure 1-year AF-free rate of $69.9 \%$ to $76.7 \%$ for paroxysmal AF and $41.9 \%$ to $48.4 \%$ for persistent AF [29]. These numbers do not suggest that the cryoballoon will dramatically increase the success of AF ablation. In this era of economic constraints, new technologies must be evaluated carefully for contributions to impoved safety and/or efficacy of AF ablation as virtually all increase procedural costs.

$\mathrm{AF}$ ablation improves the quality of life in a high percentage of patients undergoing the procedure. Although AF ablation currently has a high rate of success [9-16], it would be desirable to eliminate AF in an even greater number of patients and to decrease the need for repeat ablations [8]. In order to incrementally improve ablation outcomes, we need a better understanding of AF. The diversity of potential AF mechanisms and our lack of understanding of how AF occurs in individual patients are summarized in an editorial by Katritsis and Camm [30]. We need new and improved methods to identify the exact AF triggers and perpetuators in each patient. For example, it might be useful to have ambulatory body surface mapping to document the site of origin of $\mathrm{AF}$ in ambulatory patients prior to ablation. This might allow us to target a particular PV or a non-PV trigger causing $\mathrm{AF}$ in an individual patient with less widespread ablation. We might also discover that individual patients have multiple initiators and that the mechanism of AF can change over time. It is likely that the best immediately available technique for improving ablation outcome is for patients to have ablation earlier in the disease process before they develop LA dilatation, fail numerous AADs [31], and progress from $\mathrm{AF} 1$ to $\mathrm{AF} 2$ or $\mathrm{AF} 3$.

\subsection{Limitations}

Our data are from a single referral center case series which has inherent limitations including patient selection and referral bias. It is difficult to determine how much of our temporal reduction in complications was due to improved operator skill vs. procedural modifications over time, such as lowering our target ACT [18] or utilizing the RF transseptal needle [20]. As in all long-term follow-up studies, the ability to monitor patients decreased over time and we could have missed asymptomatic $\mathrm{AF}$ despite our regular follow-up and frequent contact by trained research personnel. Despite its shortcomings, our method of long-term follow-up is essentially the same as that used by most other referral centers reporting on long-term outcomes of AF ablations [11, 12, 26, 32]. Any missed asymptomatic AF should have occurred equally across all years and is not likely to have changed the conclusions of our study.

\section{Conclusions}

The AF ablation procedure has progressed over the last decade and, based on our experience, can be done increasingly faster and more safely, with shorter fluoroscopy and procedure times than at its inception. Despite these procedural advances, AF ablation appears to have plateaued with regard to our ability to eliminate AF in a higher percentage of patients. New technologies such as robotic and magnetic navigation systems and cryoballoon ablation systems do not seem to dramatically improve current ablation outcomes, as they only allow us to do what we are already doing with a different tool, rather than help us decide what we should be ablating in each individual patient. Breaking through the ceiling on AF ablation efficacy that we reached several years ago will probably require improved understanding of the mechanism of AF initiation and maintenance in individual patients in order to personalize ablation protocols to each patient's needs. The precise pathway to this new paradigm is a bit unclear but perhaps new technology that reveals the mechanisms of AF rather than technology designed to ablate AF might lead us there more rapidly.

Acknowledgments Patricia Barberini, R.N., Cynthia Lebsack, Pharm.D, Alfie Pierantoni, William Fleming, and Glenda Rhodes assisted with data and manuscript management.

Disclosures Dr. Mead is a member of the advisory board of Medtronic, iRhythm, and Proteus Biomedical; a stockholder at iRhythm and Proteus Biomedical; and a Director of iRhythm and Voyage Medical. Drs. Winkle and Patrawala are investigators of Cardio Robotics and CABANA Trial. Dr. Patrawala is a consultant and stockholder of Voyage Medical and a speaker of St. Jude Medical. Dr. Engel is a speaker of Medtronic. Dr. Kong is a member of the advisory board of Medtronic and Biotronik. All authors are investigators of Medtronic/Sanofi-Aventis. 
Open Access This article is distributed under the terms of the Creative Commons Attribution Noncommercial License which permits any noncommercial use, distribution, and reproduction in any medium, provided the original author(s) and source are credited.

\section{References}

1. Haissaguerre, M., Jais, P., Shah, D., Takahashi, A., Hocini, M., Quiniou, G., Garrigue, S., Mouroux, A. L., Metayer, P. L., \& Clementy, J. (1998). Spontaneous initiation of atrial fibrillation by ectopic beats originating from the pulmonary veins. The New England Journal of Medicine, 339, 659-666.

2. Lin, W.-S., Tai, C.-T., Hsieh, M.-H., Tsai, C.-F., Lin, Y.-K., Tsao, H.-M., Huang, J.-L., Yu, W.-C., Yang, S.-P., Ding, Y.-A., Chang, M.-S., \& Chen, S.-A. (2003). Catheter ablation of paroxysmal atrial fibrillation initiated by non-pulmonary vein ectopy. Circulation, 107, 3176-3183.

3. Katritsis, D. G., Giazitzoglou, E., Zografos, T., Pokushalov, E., Po, S. S., \& Camm, A. J. (2011). Rapid pulmonary vein isolation combined with autonomic ganglia modification: A randomized study. Heart Rhythm, 8, 672-678.

4. Pokushalov, E., Romanov, A., Shugayev, P., Artyomenko, S., Shirokova, N., Turov, A., \& Katritsis, D. G. (2009). Selective ganglionated plexi ablation for paroxysmal atrial fibrillation. Heart Rhythm, 6, 1257-1264.

5. Nademanee, K., McKenzie, J., Kosar, E., Schwab, M., Sunsaneewitayakul, B., Vasavakul, T., Khunnawat, C., \& Ngarmukos, T. (2004). A new approach for catheter ablation of atrial fibrillation: Mapping of the electrophysiologic substrate. Journal of the American College of Cardiology, 43, 2044-2053.

6. Chou, C. C., Chang, P. C., Wen, M. S., Lee, H. L., Chane, T. C., Yerh, S. J., \& Wu, D. (2011). Epicardial ablation of rotors suppressed inducibility of acetylcholin-induced atrial fibrillation in left pulmonary vein-left atrium preparations in a beagle heart failure model. Journal of the American College of Cardiology, $58,158-166$.

7. Calkins, H., Brugada, J., Packer, D. L., Capppato, R., Chen, S., Crijns, H., Damiano, R. J., Davies, D. W., Haines, D. E., Haissaguerre, M., Iesaka, Y., Jackman, W., Jais, P., Kottkamp, H., Kuck, K. H., Lindsay, B. D., Marchlinski, F. E., McCarthy, P. M., Mont, J. L., Morady, F., Nademanee, K., Natale, A., Pappone, C., Prystowsdy, E., Raviele, A., Ruskin, J. N., \& Shemin, R. J. (2007). HRS/EHRA/ECAS expert consensus statement on catheter and surgical ablation of atrial fibrillation: Recommendations for personnel, policy, procedures and follow-up. Heart Rhythm, 4, 1-46.

8. Winkle, R. A., Mead, R. H., Engel, G., \& Patrawala, R. A. (2011). Long term results of atrial fibrillation ablation: The importance of all initial ablation failures undergoing a repeat ablation. American Heart Journal, 162, 193-200.

9. Shah, A. N., Mittal, S., Sichrovsky, T. C., Cotiga, D., Arshad, A., Maleki, K., Pierce, W. J., \& Steinberg, J. S. (2008). Long-term outcome following successful pulmonary vein isolation: Pattern and prediction of very late recurrence. Journal of Cardiovascular Electrophysiology, 19, 661-667.

10. Gerstenfeld, E. P., Sauer, W., Callans, D. J., Dixit, S., Lin, D., Russo, A. M., Beldner, S., McKernan, M., \& Marchlinski, F. E. (2006). Predictors of success after selective pulmonary vein isolation of arrhythmogenic pulmonary veins for treatment of atrial fibrillation. Heart Rhythm, 3, 165-170.

11. Tzou, W. S., Marchlinski, F. E., Zado, E. S., Lin, D., Dixit, S., Callans, D. J., Cooper, J. M., Bala, R., Garcia, F., Hutchinson, M. D., Riley, M. P., Verdino, R., \& Gerstenfeld, E. P. (2010). Longterm outcome after successful catheter ablation of atrial fibrillation. Circulation. Arrhythmia and Electrophysiology, 3, 237-242.
12. Wokhlu, A., Hodge, D. O., Monahan, K. H., Asirvatham, S. J., Friedman, P. A., Munger, T. M., Cha, Y. M., Win-Kuang, S., Brady, P. A., Bluhm, C. M., Haroldson, J. M., Hammill, S. C., \& Packer, D. L. (2010). Long-term outcome of atrial fibrillation ablation: Impact and predictors of very late recurrence. Journal of Cardiovascular Electrophysiology, 21, 1071-1076.

13. Patel, D., Mohanty, P., Di Biase, L., Sanchez, J. E., Shaheen, M. H., Burkhardt, J. D., Bassouni, M., Cummings, J., Wang, Y., Lewis, W. R., Diaz, A., Horton, R. P., Beheiry, S., Hongo, R., Gallinghouse, G. J., Zagrodzky, J. D., Bailey, S. M., Al-Ahmad, A., Wang, P., Schweikert, R. A., \& Natale, A. (2010). Outcomes and complications of catheter ablation for atrial fibrillation in females. Heart Rhythm, 7, 167-172.

14. Bhargava, M., Di Biase, L., Mohanty, P., Prasad, S., Martin, D. O., Andrew, M. W., Wazni, O. M., Burkhardt, J. D., Cummings, J. E., Khaykin, Y., Verma, A., Hao, S., Beheiry, S., Hongo, R., Rossillo, A., Raviele, A., Bonso, A., Themistoclakis, S., Stewart, K., Saliba, W. I., Schweikert, R. A., \& Natale, A. (2009). Impact of type of atrial fibrillation and repeat catheter ablation on long-term freedom of atrial fibrillation: Results from a multicenter study. Heart Rhythm, 6, 1403 1412.

15. Katritsis, D., Wood, M. A., Giazitzoglou, E., Shepard, R. K., Kourlaba, G., \& Ellenbogen, K. A. (2008). Long-term follow-up after radiofrequency catheter ablation for atrial fibrillation. Europace, 10, 419-424.

16. Forleo, G. B., Tondo, C., De Luca, L., Dello Russo, A., Casella, M., De Sanctis, V., Clementi, F., Fagundes, R. L., Leo, R., Romeo, F., \& Mantica, M. (2007). Gender-related differences in catheter ablation of atrial fibrillation. Europace, 9, 613-620.

17. Piccini, J. P., Lopes, R. D., Kong, M. H., Hasselblad, V., Jackson, K. J., \& Al-Khatib, S. M. (2009). Pulmonary vein isolation for the maintenance of sinus rhythm in patients with atrial fibrillation: A meta-analysis of randomized controlled trials. Circulation. Arrhythmia and Electrophysiology, 2, 626-633.

18. Winkle, R. A., Mead, R. H., Engel, G., \& Patrawala, R. A. (2011). Safety of lower activated clotting times during atrial fibrillation ablation using open irrigated tip catheters and a single transseptal puncture. The American Journal of Cardiology, 107, 704-708.

19. Winkle, R. A., Mead, R. H., Engel, G., \& Patrawala, R. A. (2011). Relation of early termination of persistent atrial fibrillation by cardioversion or drugs to ablation outcomes. The American Journal of Cardiology, 108, 374-379.

20. Winkle, R. A., Mead, R. H., Engel, G., \& Patrawala, R. A. (2011). The use of a radiofrequency needle improves the safety and efficacy of transseptal puncture for atrial fibrillation ablation. Heart Rhythm, 8, 1411-1415.

21. Winkle, R. A., Mead, R. H., Engel, G., \& Patrawala, R. A. (2011). Atrial fibrillation ablation: Perpetual motion of open irrigated tip catheters at 50 watts is safe and improves outcomes. Pacing Clin Elecrophysiol, 34, 531-539.

22. Winkle RA, Mead RH. Engel G, Kong MH, Patrawala RA (2012). The use of dabigitran immediately following atrial fibrillaton ablation. $J$ Cardiovasc Electrophysiol (in press)

23. Dibiase, L., Conti, S., Mohanty, P., Bai, R., Sanchez, J., Walton, D., John, A., Santangeli, P., Elayi, C. S., Beheiry, S., Gallinghouse, G. J., Mohanty, S., Horton, R., Bailey, S., Burkhardt, J. D., \& Natale, A. (2011). General anesthesia reduces the prevalence of pulmonary vein reconnection during repeat ablation when compared with conscious sedation: Results from a randomized study. Heart Rhythm, 8(3), 368-372.

24. Lemola, K., Hall, B., Cheung, P., Good, E., Han, J., Tamirisa, K., Chugh, A., Bogun, F., Pelosi, F., Morady, F., \& Oral, H. (2004). Mechanisms of recurrent atrial fibrillation after pulmonary vein isolation by segmental ostial ablation. Heart Rhythm, 1, 197-202. 
25. Stabile, G., Turco, P., La Rocca, V., Nocerino, P., Stabile, E., \& De Simone, A. (2003). Is pulmonary vein isolation necessary for curing atrial fibrillation? Circulation, 108, 657-660.

26. Weerasooriya, R., Khairy, P., Litalien, J., Macle, L., Hocini, M., Sacher, F., Lellouche, N., Knecht, S., Wright, M., Nault, I., Miyazaki, S., Scavee, C., Clementy, J., Haissaguerre, M., \& Jais, P. (2011). Catheter ablation for atrial fibrillation. Are results maintained at 5 years of follow-up? Journal of the American College of Cardiology, 57, 160-166.

27. Miyazaki, S., Shah, A. J., Xhaët, O., Derval, N., Matsuo, S., Wright, M., Nault, I., Forclaz, A., Jadidi, A. S., Knecht, S., Rivard, L., Liu, X., Linton, N., Sacher, F., Hocini, M., Jaïs, P., \& Haïssaguerre, M. (2010). Remote magnetic navigation with irrigated tip catheter for ablation of paroxysmal atrial fibrillation. Circulation. Arrhythmia and Electrophysiology, 3, 585-589.

28. Hlivák, P., Mlčochová, H., Peichl, P., Čihká, R., Wichterle, D., \& Kautzner, J. (2011). Robotic navigation in catheter ablation for paroxysmal atrial fibrillation: Midterm efficacy and predictors of postablation arrhythmia recurrences. Journal of Cardiovascular Electrophysiology, 22, 534-540.

29. Andrade, J. G., Khairy, P., Guerra, P. G., Deyell, M. W., Rivare, L., Macle, L., Thibaule, B., Talajic, M., Roy, D., \& Dubuc, M. (2011). Efficacy and safety of cryoballoon ablation for atrial fibrillation: A systematic review of published studies. Heart Rhythm, 8, 1444-1451.

30. Katritsis, D. G., \& Camm, A. J. (2007). Catheter ablation of atrial fibrillation: Do we know what we are doing? Europace, 9, 1002-1005.

31. Winkle RA, Mead RH. Engel G, Kong MH, Patrawala RA (2012). Prior antiarrhythmic drug use and the outcome of atrial fibrillation ablation. Europace (in press)

32. Cheema, A., Vasamraddy, C. R., Dalal, D., Marine, J. E., Dong, J., Henrikson, C. A., Spragg, D., Cheng, A., Nazarian, S., Sinha, S., Halperin, H., Berger, R., \& Calkins, H. (2006). Long-term single procedure efficacy of atrial fibrillation ablation. Journal of Interventional Cardiac Electrophysiology, 15, 145-155. 